\title{
Interacting bubble clouds and their sonochemical production
}

\author{
Laura Stricker \\ Physics of Fluids Group, Impact Institute \& Burgers Center for Fluid Dynamics, \\ University of Twente, \\ Drienerlolaan 5, \\ 7500 AE Enschede, The Netherlands
}

\author{
Benjamin Dollet \\ Institut de Physique de Rennes, \\ UMR 6251 CNRS/Université de Rennes 1,
} Bâtiment 11A, Campus Beaulieu, Avenue du Général Leclerc 263 35042 Rennes Cedex, France

David Fernández Rivas Mesoscale Chemical Systems Group, MESA+ Research Institute, University of Twente, Drienerlolaan 5, 7500 AE Enschede, The Netherlands

\author{
Detlef Lohse \\ Physics of Fluids Group, Impact Institute \& Burgers Center for Fluid Dynamics, \\ University of Twente, \\ Drienerlolaan 5, \\ 7500 AE Enschede, The Netherlands
}

(Dated: July 18, 2018) 


\begin{abstract}
Acoustically driven air pockets trapped in artificial crevices on a surface can emit bubbles which organize in (interacting) bubble clusters. With increasing driving power Fernández Rivas et al. [Angew. Chem. Int. Ed., 2010] observed three different behaviors: clusters close to the very pits out of which they had been created, clusters pointing toward each other, and merging clusters. The latter behavior is highly undesired for technological purposes as it is associated with a reduction of the radical production and an enhancement of the erosion of the reactor walls. The dependence on the control parameters such as the distance of the pits and the conditions for cluster-merging are examined. The underlying mechanism, governed by the secondary Bjerknes forces, turns out to be strongly influenced by the nonlinearity of the bubble oscillations and not directly by the number of nucleated bubbles. The Bjerknes forces are found to dampen the bubble oscillations, thus reducing the radical production. Therefore, the increased number of bubbles at high power could be the key to understand the experimental observation that, after a certain power threshold, any further increase of the driving does not improve the sonochemical efficiency.
\end{abstract}

PACS numbers: 4335Vz, 4335Ei 


\section{INTRODUCTION}

Sonochemistry is the use of ultrasound to achieve a chemical conversion. Imploding microbubbles can produce localized extreme temperature and pressure conditions. As a result, high energy chemical conversions can be triggered, eventually resulting in the production of highly reactive radical species $\underline{-1}-\underline{-6}$. The applications of these reaction products are manifold, including synthesis of fine chemicals, food ingredients and pharmaceuticals, degradation of water contaminants $\underline{\underline{7}-\underline{10}}$, textile processing $\underline{\underline{11}}$ and cell disruption $\underline{\underline{12}}$. Fernández Rivas et al $\stackrel{13,14}{\underline{14}}$ have recently proposed an efficient way to do sonochemistry, controlling cavitation by using micropits grooved on silicon substrates, following the original idea of Bremond et al $\underline{\underline{15}}$. At sufficient acoustic pressures, a bubble cluster is generated in the liquid above each pit. If two pits or more are present, such clusters tend to attract and merge over a certain pressure amplitude. It has been shown that merging clusters are associated to a reduction in the radical production respect to the case of not interacting clusters $\underline{14}$ and erosion of the reactor walls $\underline{\underline{16}}$. Therefore, in efficient sonochemical reactors design, one should maximize the number of pits (i.e. of bubbles) but should avoid cluster-merging conditions. The goal of the present work is to understand the transition between the three possible behaviors observed in experiments (see Fig. 1): individual clusters next to the pit out of which they were generated (behavior 1), individual clusters pointing towards the central array of the pits (behavior 2), and clusters migrating towards the center (behavior 3). The key factor to study these phenomena are the acoustic interactions between different bubbles, namely the secondary Bjerknes forces 17,18 .

Though these forces have been largely investigated both for bubble pairs $\underline{19}-\underline{24}$ and for bubble clouds 5,26 , making an a-priori prediction even on their sign is a non-trivial matter. The linear theory predicts that two acoustically driven bubbles oscillate in phase and attract each other when the driving frequency is greater or lower than both their resonance frequencies, while they oscillate in counter-phase and repel with a driving fre- 
quency in-between their resonance frequencies ${ }^{27}$. Thus, Bjerknes forces are expected to be attractive for bubbles of equal size $\mathrm{e}^{22,23}$. As a first, qualitative statement, we can therefore expect the cluster-cluster interaction to be attractive (since the pits are identical, the bubbles of each cluster should have similar sizes). Hence, the fact that clusters merge only above a certain threshold suggests that the pit-cluster interaction is also attractive, and that the cluster-cluster interaction must overcome the pit-cluster one to achieve cluster merging. However, it has been proved by a number of authors that the sign of the Bjerknes forces can be reversed due to several mechanisms neglected by the classical linear theory, such as the effect of secondary harmonics 21,24 , the resonance-like behavior of small bubbles (below their resonance size) near the dynamic Blake threshold 28 and viscous effects during translational motion $\underline{29}$. Several studies of two bubbles interacting in a strong acoustic field have also shown that bubbles oscillating nonlinearly can form a bound pair with a steady spacing rather than collide and coalesce, as linear Bjerknes theory would predict $\underline{30}-\underline{33}$. Therefore caution is required when one wishes to understand the behavior of interacting clusters of bubbles.

In the present work we will investigate the Bjerknes forces acting upon the clusters and their dependence on different parameters, such as the size and the number of the bubbles, the size of the clusters, the distance between the pits and the driving pressure. We will show the influence of these forces upon the bubble dynamics and the radical production and will address the conditions required for the different transitions, thus providing practical indications for efficient sonochemical sonoreactors design, where one wishes to have the highest possible number of non-interacting micropits.

\section{MODEL}

In the experiment of Ref. $\underline{\underline{14}}$, the bubble population is quite polydisperse. In top view, clusters appear as diffuse circles of radius $R_{c}$ (Fig. 1); a side view reveals that they are in contact with the substrate and have roughly an elliptic shape (Fig. 7 of Ref. $\underline{\underline{14}}$ ) with 
a long axis parallel to the substrate, and not much larger than the short axis. To simplify the problem, we will assume that each cluster is a sphere of radius $R_{c}$ in tangential contact with the substrate and we will neglect polydispersity. We will consider $R_{c}$ as time-invariant for simplicity reasons, although in reality this holds only on average, for a give applied power, but not in a rigorous sense. We will also assume that all the bubbles have the same radius $R(t)$ and that the two cluster have the same number of bubbles $N$, constant in time.

Each individual bubble in a cluster experiences acoustic interactions from the pits, from the neighboring bubbles of the same cluster, from the bubbles of the other cluster, and from all image bubbles, given the presence of the hard silicon substrate (see Fig. 2). We describe the behavior of a single bubble belonging to one of the clusters by extending the model previously developed in Refs $\underline{\underline{34}} \underline{\underline{36}}$ and validated in $\frac{37}{\underline{7}}$, to incorporate the effect of the secondary Bjerknes forces upon the pulsation of the bubble. This model is then coupled with a static force balance in order to study the switch between the different conditions observed experimentally (see Fig. 1).

The model that we adopt for the single bubble is an ODE model based on the assumptions that the gas inside the bubble is a perfect gas and that the bubble has a uniform temperature and pressure. The temperature evolution is derived from the energy equation. Heat and mass transfer are treated with a boundary layer approximation $\underline{34}-\underline{36}$. Evaporation/condensation phenomena are kept into account, as well as the variation of the transport parameters due to compositional changes of the mixture. A list of 45 chemical reactions is included, with their temperature dependent chemical kinetics, governed by Arrhenius law. We refer the reader to $\underline{\underline{36}}$ for a detailed description of these parts of the model, and we concentrate in the following upon the treatment of the Bjerknes forces.

The radial dynamics of a bubble belonging to cluster 1 is described by means of a modified Rayleigh-Plesset equation, keeping into account the effect of the secondary 
Bjerknes forces on the radial pulsation ${ }^{26,38}$ :

$$
\begin{aligned}
(1- & \left.\frac{\dot{R}}{c}\right) R \ddot{R}+\frac{3}{2}\left(1-\frac{\dot{R}}{3 c}\right) \dot{R}^{2} \\
& =\frac{1}{\rho}\left(1+\frac{\dot{R}}{c}\right)\left(p-p_{\infty}-P(t)\right)+\frac{R \dot{p}}{\rho c}-\frac{4 v \dot{R}}{R}-\frac{2 \sigma}{\rho R}-T_{B j} .
\end{aligned}
$$

Here the dots are used for time derivatives, $R$ is the radius of the bubble, $c$ is the speed of sound, $\rho$ is the density of the liquid, $v$ its kinematic viscosity, $\sigma$ is the surface tension, $p_{\infty}$ the static pressure and $P(t)=P_{a} \cos \omega t$ is the acoustic driving pressure, with $P_{a}$ the driving amplitude, $f=\omega / 2 \pi$ the frequency and $\tau=1 / f$ the period of the driving. $T_{B j}$ is a coupling term expressing the effect of the interaction with the other bubbles, both real and imaginary, and the pits

$$
T_{B j}=T_{c 1}+T_{c 2}+T_{m 1}+T_{m 2}+T_{p 1}+T_{p 2}
$$

$T_{c 1}$ and $T_{c 2}$ are the coupling terms with the bubbles of the same cluster and the other cluster respectively, $T_{m 1,2}$ are the coupling terms with the two mirror clusters, $T_{p 1,2}$ are the coupling terms with the two pits.

The coupling term $T_{2 \rightarrow 1}$ between two isolated bubbles, describing the influence of bubble 2 on the radial oscillations of bubble 1 , can be written as 28

$$
T_{2 \rightarrow 1}=\frac{1}{d}\left(R_{2}^{2} \ddot{R}_{2}+2 R_{2} \dot{R}_{2}^{2}\right)
$$

Therefore, the coupling term between one bubble $i$ and the other bubbles of the cloud to which it belongs becomes

$$
T_{c 1}=\sum_{j \neq i} \frac{R_{j}^{2} \ddot{R}_{j}+2 R_{j} \dot{R}_{j}^{2}}{r_{i j}},
$$

in which $r_{i j}$ is the distance between bubbles $j$ and $i$. Following the approximation of Yasui et al. $\underline{\underline{26}}$, i.e. neglecting polydispersity and assuming that the cluster has constant density, we get:

$$
T_{c 1} \simeq 2 \pi n R_{c}^{2}\left(R^{2} \ddot{R}+2 R \dot{R}^{2}\right)
$$

where $R_{c}$ is the cluster radius and $n$ is the number density of bubbles, $n \simeq 3 N / 4 \pi R_{c}^{3}$. 
The coupling term between the considered bubble of cluster 1 and all the bubbles of cluster 2 is expressed as in Ref. $\frac{26}{\underline{6}}$

$$
T_{c 2} \simeq \frac{N}{d_{c}}\left(R^{2} \ddot{R}+2 R \dot{R}^{2}\right),
$$

where $d_{c}$ is the distance between the two clouds. This is valid as long as $d_{c} \gg R_{c}$. The coupling terms $T_{m 1,2}$ with the two mirror clusters are expressed in a similar way.

In order to model the interaction of the bubble with the pit, we consider the pit as an effective bubble with the same resonant frequency $\omega_{p}$ and damping coefficient $\beta$ as the pit. Although the pit is not a spherical bubble, and may oscillate in a nonlinear fashion, we will treat it as a harmonic oscillator. This assumption is acceptable as long as we showed in Ref $\frac{39}{\underline{9}}$ that, in the considered parametric range, large amplitude oscillations of a gas pocket entrapped inside a cylindrical pit present an overall behavior similar to small amplitude oscillations, with a slightly lower damping but the same resonance frequency. Hence, we consider the pit like a spherical bubble of radius at rest $R_{p}^{0}$, such that $\omega_{p}^{2}=3 p_{\infty} / \rho\left(R_{p}^{0}\right)^{2}$, experiencing linear oscillations $\stackrel{40}{ }: R_{p}=R_{p}^{0}\left(1+x_{p}\right)$ with:

$$
\ddot{x}_{p}+2 \beta \dot{x}_{p}+\omega_{p}^{2} x_{p}=-\frac{p_{a}(t)}{\rho\left(R_{p}^{0}\right)^{2}} .
$$

The values of $\omega_{p}$ and $\beta$ are taken from the recent results of Gelderblom et al. $\underline{\underline{41}}$. These authors have computed the acoustic response of a gas pocket entrapped in a pit, providing its eigenfrequency and damping coefficient, in two limits: potential flow, and unsteady Stokes equation. For a cylindrical pit of radius $a$ and height $h$, the results mainly depend on the parameter $P=\kappa a^{2} p_{0}^{\infty} / h \sigma$. In the experiments of ${ }^{13,14}, a=15 \mu \mathrm{m}$, $h=10 \mu \mathrm{m}, p_{0}^{\infty}=10^{5} \mathrm{~Pa}$ and $\sigma=0.07 \mathrm{~N} / \mathrm{m}$. The temperature was controlled providing an isothermal behavior within a precision of $1 \mathrm{~K} \underline{\underline{14}}$ and therefore $\kappa=1$. Hence we compute $P=32$, from which $\hat{\omega}_{p}=5.82$ and $\hat{\beta}=0.26$ in the Stokes regime, and $\hat{\omega}_{p}=6.04$ and $\hat{\beta}=0.20$ in the potential regime. The dimensionless frequency is defined as $\hat{\omega}_{p}=\omega_{p} \sqrt{\rho a^{3} / \sigma}$, with a rescaling angular frequency $\sqrt{\sigma / \rho a^{3}}=1.46 \times 10^{5} \mathrm{rad} / \mathrm{s}$. Hence, taking $\hat{\omega}_{p}=6$, the resonance frequency of a pit equals $f_{p}=143 \mathrm{kHz}$, with 
$f_{p}=\omega_{p} / 2 \pi$. In the experiments of ${ }^{13}, \underline{14}$, it is not clear which of the two regimes, potential or Stokes, applies best, but it is seen that the numerical values of eigenfrequency and damping differ by only $10 \%$. Moreover, in the numerical work of Ref $\frac{39}{\underline{3}}$ it was shown that in the intermediate regime where both inertia and viscosity are present, the overall behavior of the pit is closer to the Stokes regime, when the static pressure equals $p_{\infty}=$ $1 \mathrm{~atm}$. So, we use hereby the results related to the Stokes regime for the damping and we take $\beta=3.8 \times 10^{5} \mathrm{~s}^{-1}$.

By substituting $R_{p}(t)$ inside (3), the coupling term between the considered bubble of cluster 1 and pit 1 can be expressed as

$$
T_{p 1}=\frac{1}{d_{p 1}}\left(R_{p}^{0}\right)^{3}\left(1+x_{p}\right)\left(2 \dot{x}_{p}^{2}+x_{p} \ddot{x}_{p}+\ddot{x}_{p}\right),
$$

where $d_{p 1}$ is the distance between the bubble and the pit. The coupling term $T_{p 2}$ between the bubble of cluster 1 and pit 2 is expressed correspondingly.

We now turn to the forces experienced by the clusters (see Fig.2). As we want to study the transition between behavior 1 and behavior 2, we will focus on the forces acting on the horizontal plane. Hence, we will neglect both the buoyancy and the primary Bjerknes force, which is directed in the vertical direction, because the driving pressure is a standing wave with a anti-node on the substrate and a node at the free water-air surface $\stackrel{42}{\text {. }}$.

In a static equilibrium condition, all the forces acting upon a bubble of cluster 1 with an horizontal component are secondary Bjerknes forces. Such forces are exerted by the other cluster $\vec{F}_{c}$, by the mirror clusters $\vec{F}_{m 1,2}$ and by the pits $\vec{F}_{p 1,2}$. In order to derive them, we first consider two oscillating bubbles, of volume $\mathcal{V}_{1}$ and $\mathcal{V}_{2}$, separated by a distance $d$ much greater than their radii; then bubble 1 experiences a force equal to $\underline{28}$ :

$$
\vec{F}_{2 \rightarrow 1}=\frac{\rho \ddot{\mathcal{V}}_{2} \mathcal{V}_{1}}{4 \pi d^{2}} \hat{e}_{2 \rightarrow 1}
$$

with $\hat{e}_{2 \rightarrow 1}$ the unit vector pointing from bubble 2 to bubble 1 . For bubbles of the same radius $R$, this reduces to $\vec{F}_{2 \rightarrow 1}=4 \pi \rho R^{3}\left(R^{2} \ddot{R}+2 R \dot{R}^{2}\right) \hat{e}_{2 \rightarrow 1} / 3 d^{2}$. Let us first notice that the forces between bubbles pertaining to the same cluster are responsible of the cluster 
cohesion, but are irrelevant to the interaction between different clusters; hence, we neglect them to assess the stability of behavior 1 . Assuming that $R_{c} \ll d_{c}$, each bubble of cluster 1 experiences from cluster 2 a force equal to $\underline{26,38}$ :

$$
\vec{F}_{c}=\frac{4 \pi \rho N R^{3}\left(R^{2} \ddot{R}+2 R \dot{R}^{2}\right)}{3 d_{c}^{2}} \hat{e}_{2 \rightarrow 1} .
$$

As $d_{c}=d-2 \delta$ (Fig. 2), the horizontal component of $\vec{F}_{c}$ is given by

$$
F_{c, x}=\frac{A_{c}}{(d-2 \delta)^{2}}
$$

with $A_{c}=4 \pi \rho N R^{3}\left(R^{2} \ddot{R}+2 R \dot{R}^{2}\right) / 3$. The forces acting on each bubble of cluster 1 from the mirror clusters, namely $\vec{F}_{m 1}$ and $\vec{F}_{m 2}$, and their horizontal components can be expressed in a similar way.

The secondary Bjerknes force acting over each bubble of cluster 1 from pit 1 is found by substituting the volume of the equivalent bubble corresponding to the pit $\mathcal{V}_{p}=4 \pi R_{p}^{3} / 3$ into Eq. (9),

$$
\vec{F}_{p 1}=\frac{4}{3} \frac{\pi \rho\left(R_{p}^{0}\right)^{3}}{d_{p}^{2}} R^{3}\left(1+x_{p}\right)\left[2 \dot{x}_{p}^{2}+\ddot{x}_{p}\left(1+x_{p}\right)\right] \hat{e}_{p} .
$$

Here $\hat{e}_{p}$ is the unit vector pointing from the pit to the bubble and $d_{p}^{2}=h_{p}^{2}+\delta^{2}$, with $h_{p}=$ $R_{c}+h / 2$. Even if $d_{p}$ depends on the location of the bubble within its cluster, we will take $d_{p}$ as the distance between the pit and the center of the cluster; in practice, off-centered bubbles within the cluster will experience pit interaction of a different magnitude, but this will be compensated by the interaction with the other bubbles responsible of the cohesion of the cluster. Given $\hat{e}_{p} \cdot \hat{e}_{x}=\delta / d_{p 1}$, the horizontal component of $F_{p 1}$ can be calculated from (12) as

$$
F_{p 1, x}=A_{p 1} \frac{\delta}{\left[h_{p}^{2}+\delta^{2}\right]^{3 / 2}}
$$

with $A_{p 1}=4 \pi \rho\left(R_{p}^{0}\right)^{3} R^{3}\left(1+x_{p}\right)\left[2 \dot{x}_{p}^{2}+\ddot{x_{p}}\left(1+x_{p}\right)\right] / 3$. The force $F_{p 2}$ acting on each bubble of cluster 1 from pit 2 and its horizontal component are calculated similarly. 


\section{RESULTS}

\section{A. Transition from individual clusters to merging clusters}

Fernández Rivas et al $\stackrel{14}{\underline{1}}$ provide an experimental characterization of the number and size of the bubbles present in the cluster for one, two and three pits, and for three different powers. They show that the number of bubbles $N$ increases at increasing power, and that both the average bubble radius $(R \simeq 10 \mu \mathrm{m})$ and the most probable radius $(R \simeq 3 \mu \mathrm{m})$ have no significant dependence on the power and on the number of pits. According to (10) and (12), at first order, i.e. neglecting the effect of the other bubbles on $R(t)$, the cluster-cluster force is proportional to $N$, whereas the pit-cluster force does not depend on $N$. The threshold pressure for merging may thus originate either from the fact that $N$ increases at increasing power or from higher order effects of bubble oscillations on the secondary Bjerknes forces, contained inside the terms $A_{p}$ and $A_{c}$. In order to investigate the transitions between the three different behaviors found in experiments (see Fig. (1), we adopt a quasi-static "adiabatic" approach: we take $\delta$ as a constant over time, representing the displacement of the bubble and therefore of the cloud from the initial equilibrium position. At each instant, we calculate the horizontal components of $\vec{F}_{c}, \vec{F}_{p 1,2}$ and $\vec{F}_{m 1,2}$. We stop the calculation after one cycle, to match the experimental conditions of Ref. $\underline{\underline{14}}$, where the bubbles did not survive after the first collapse. We perform the time averages of the forces over the whole cycle and we verify whether the following holds

$$
\left\langle F_{x}^{*}\right\rangle>\left\langle F_{p 1, x}\right\rangle
$$

where $\left\langle F_{x}^{*}\right\rangle=\left\langle F_{c}\right\rangle+\left\langle F_{p 2, x}\right\rangle+\left\langle F_{m 2, x}\right\rangle$ is the sum of the forces attracting the clusters towards each other and $\langle\cdot\rangle$ denotes the time average over the first acoustic cycle.

In order to study the transition between behavior 1 and behavior 2, i.e. the inception of the motion, we consider a small initial horizontal displacement $\delta=R_{0}$ of the bubble from the pit axes and therefore from its rest conditions. The motion starts once Eq. (14) holds. However, this does not imply that the bubble will eventually reach the center of 
the pit array, as we will show below. An example of forces acting upon a bubble with an initial displacement of $\delta=R_{0}$ is given on Fig. 3, Sign inspection shows that the clustercluster forces both with the real and the mirror cluster 2 are always attractive, while the pit-clusters forces become repulsive at high driving pressure. Above pressures of $150 \mathrm{kPa}$ the noise increases, probably due to the nonlinearity of the problem.

In order to study the transition between behavior 2 and behavior 3, i.e. the merging of the clusters, we consider both $F_{x}^{*}$ and $F_{p 1, x}$ as a function of $\delta$. The transition occurs once Eq. (14) holds at all $\delta$. As a function of $\delta,\left\langle F_{p 1, x}\right\rangle$ has a maximum, generally (but not always) corresponding to the point $\bar{\delta}$ where the pit traps the cluster. The transition is graphically sketched on Fig. 4. The black lines correspond to merging clusters (behavior 3); the lit-gray lines correspond to the situation where $\left\langle F_{x}^{*}\right\rangle$ is strong enough to induce the inception of the motion but too weak to overcome the barrier constituted by the restoring force of the pit. Therefore the cluster remains attached to its pit, with a small displacement given by the intersection of the two curves (behavior 2). The mid-gray lines represent the transition between behavior 2 and behavior 3 , and the corresponding pressure amplitude will be denoted from now on as $\bar{P}_{a}$.

In Fig. 5 we plot the driving pressure required for the two transitions, namely the one for cluster 1 to start moving (dash-dotted line) and the one to overcome the trapping force of the pit (solid line), as a function of the distance between the pits $d$. According to the experimental conditions of Ref $\underline{\underline{14}}$, we consider two clusters with $R_{c}=100 \mu \mathrm{m}$, $N=100, R_{0}=10 \mu \mathrm{m}$. As the distance between the pits increases, a higher $P_{a}$ is required for merging. For $d=1000 \mu \mathrm{m}$, we calculated that the transition occurs at $\bar{\delta}=94 \mu \mathrm{m}$, with $\bar{P}_{a}=270 \mathrm{kPa}$. In the experiments, the maximum displacement of the clusters before they detach from the pit and started coalescing was $\bar{\delta} \sim R_{c}$ (see Fig. (1). Moreover, three pressure values have been measured, corresponding to the three different levels of of the applied power (low, medium and high), $165 \mathrm{kPa}, 225 \mathrm{kPa}$ and $350 \mathrm{kPa}$ respectively. The transition occurred between $225 \mathrm{kPa}$ and $350 \mathrm{kPa} \underline{14}$. Despite the approximations of the model, such as the equal size and monodispersity of the bubbles inside the cluster and 
the time-invariance of both the number of the bubbles and the size of the clusters, we remark that these values are extremely close to the experimental ones.

Also as a function of the distance between the pits, the numerical results reproduce the same trend found in experiments: when $d$ increases, so does $\bar{P}_{a}$, until a limiting value of $d$, where no merging is possible anymore. In the experiments, this limit was found at $d$ $=1500 \mu \mathrm{m}$, in the simulation at $d=1350 \mu \mathrm{m}$. Once again, the agreement with the model is remarkably good. The trapping distance $\bar{\delta}$ increases with $d$. In Fig. 6, we show the voltage applied to the piezo when the clusters merge, at increasing applied power (black) and when the clusters detach, coming back to their own pits, at decreasing applied power

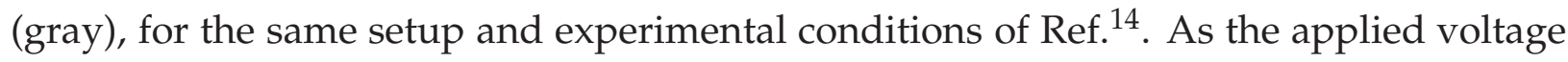
is directly connected to the driving pressure $P_{a}$, we can conclude that the driving pressure required for merging is slightly higher than the one required to detach the clusters. The theoretical investigation of this hysteretic behavior is beyond the scope of the present paper and should be addressed in future works.

For a given $R_{0}$ and $R_{c}, \bar{P}_{a}$ has a very slight dependence on the number of bubbles and increasing $N$ reduces $\bar{P}_{a}$ only until a certain value of $N$. With $R_{0}=10 \mu \mathrm{m}, R_{c}=100$ and $d=1000 \mu \mathrm{m}$ this happens for $N<50$ (see Fig. 7). For clusters with a higher number of bubbles, a further increase of $N$ does not imply a further decrease of $\bar{P}_{a}$. This means that the number of bubbles itself is not what determines the transition between separated clusters and merging clusters. Thus, we can conclude that, at medium and high power, the phenomenon is governed by nonlinear oscillations effects contained inside $A_{p}$ and $A_{c}$ in Eqs. (11) and (13).

However, the number of bubbles can still have an indirect influence on the transition: given a certain $R_{0}$ and $N$, both $\bar{P}_{a}$ and $\bar{\delta}$ are higher when the cloud radius $R_{c}$ is smaller (see Fig. (8). As nonlinear oscillating bubbles tend to form stable pairs without coalescing, i.e. the bubbles remain at a certain equilibrium distance from each other $\underline{\underline{30}} \underline{\underline{33}}$, we can expect that an increase in the number of bubbles will also lead to an increase in the cluster size and therefore to a lower $P_{a}$ at transition. 
The dependence of the pressure amplitude for clusters merging on the bubble size is non-monotonic. In a range from $R_{0}=3$ to $15 \mu \mathrm{m}$, the clusters requiring a lower $\bar{P}_{a}$ to escape from the pit region are those with $R_{0}=10 \mu \mathrm{m}$ (see Fig9). Therefore we can assume that these bubbles will also be the ones to initiate the merging. Nevertheless, the inclusion of inertia and drag, excluded from the present approach, could shift this minimum towards lower values of $R_{0}$ and it should be addressed in a future paper.

\section{B. Radical production}

In order to investigate the effects of the Bjerknes forces on the radical production, we consider a cluster with $R_{c}=100 \mu \mathrm{m}, N=100, d=1000 \mu \mathrm{m}, R_{0}=3 \mu \mathrm{m}$, driven at $f=$ $200 \mathrm{kHz}$ and $P_{a}=270 \mathrm{kHz}$. In Fig. 10 we show the radial and thermal evolution of a bubble of such a cluster. Including the Bjerknes forces (solid line) has the same effect of adding some damping to the system, as it leads to a lower expansion of the bubble $\mathrm{e}^{\underline{\underline{6}}}$ and therefore to a lower temperature at collapse respect to the case where Bjerknes forces are not included (solid-dashed line). As the radical production is related to the peak temperature through Arrhenius law, neglecting the Bjerknes forces induces a huge overestimate of the produced radicals (see Fig. 11). Moreover, the Bjerknes forces reduce the eigenfrequency of the bubble (see Fig.10), and therefore induces a further reduction of the radical production, due to the lower number of collapses per unit time respect to the isolated bubble. From the theoretical point of view this reduction of the resonance frequency can be predicted using the standard approach for the calculation of the linear resonance frequency of a bubble $43, \underline{44}$. We rewrite the modified Rayleigh-Plesset equation (1) by neglecting the effects of liquid compressibility, surface tension and viscosity, under the hypothesis of linear oscillations. Considering just the effect of the other bubbles of the same cloud the new linear frequency will be such to satisfy $\omega_{0}^{2}=3 \kappa p_{\infty} /\left[\rho R_{0}^{2}\left(1+3 / 2 N R_{0} / R_{c}\right)\right]$. With $N$ varying between 10 and $100, N R_{0} / R_{c}$ varies between 1 and 10 . For $R_{0}=10 \mu \mathrm{m}$, we compute $f_{0}=326,304,206$, and $82 \mathrm{kHz}$, respectively for $N R / R_{c}=0$ (single bubble), 1,10 , 
and 100. Given the resonance frequency of the pit $f_{p}=143 \mathrm{kHz}$ and the driving frequency $f=200 \mathrm{kHz}$, in the linear regime, i.e. at low driving amplitude, an attractive pit-cluster force is expected, in agreement with what we found. However, due to nonlinearities, at high driving amplitude, the pit-cluster force can become repulsive ${ }^{28,33}$ (see Fig. (3). In Fig. 10 and in Fig. 11 we also show the dynamic, thermal, and chemical evolution of the same bubble driven at $P_{a}=160 \mathrm{kPa}$, without the Bjerknes forces. This driving amplitude corresponds to the "effective pressure" that we calculated in our previous work for the

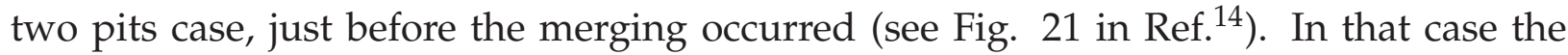
effective pressure was extracted from the experimental data by neglecting the Bjerknes forces and using the bubbles as a pressure sensor through their recorded dynamics. Although there are some differences between the radial evolution curve of a bubble driven at $P_{a}=270 \mathrm{kPa}$ undergoing Bjerknes forces and an isolated bubble driven at $P_{a}=160 \mathrm{kPa}$, the maximum and the minimum radius correspond, thus providing consistency between the present work and Ref. $\underline{\underline{14}}$.

The interaction with the other bubbles strongly influences the radical production even before the inception of the motion. In Fig. 12 we show the maximum number of $\mathrm{OH}$ radicals produced per cycle in one bubble of cluster 1 as function of the distance between the pits. When the distance between the pits decreases, so does the radical production, because the interaction with the neighboring bubbles becomes stronger, therefore damping the oscillations and decreasing the temperature at collapse. For the same reason, for a fixed number of bubbles, smaller clouds have lower radical production (Fig. 12), as the bubbles are closer. Similarly, for a fixed cluster size, raising the number of bubbles reduces the chemical production (see Fig. 13). However, in the range between 50 and 100 bubbles, there is a local maximum at $N \sim 70$. This could provide an explanation to the experimental observation that, above a certain threshold, a further increase of the applied acoustic power does not enhance the radical production $\underline{\underline{14}}$. 


\section{CONCLUSIONS}

In the present work we theoretically studied the interactions between bubbles clusters generated from ultrasonically driven silicon etched micropits $\underline{14}$. We addressed the transition between the three different behaviors observed in Ref. $\underline{\underline{14}}$ at increasing acoustic power: clusters sitting upon their own pit, clusters pointing towards each other and clusters migrating towards the center point of the pits array. We considered each cluster as a point object and we examined the secondary Bjerknes forces acting upon it. These forces depend on the displacement of the cluster from the pit. While the cluster-cluster force is always attractive, in the considered parametric range, at high driving the pit-cluster force can also become repulsive at some points very close the pit, thus favoring the inception of the motion. Given the driving frequency and the size of the bubbles and the pits, this is in contrast with the predictions of the linear theory and it has to be ascribed to the nonlinearity of the phenomenon $\underline{21,28,33}$. We found that there always exists a barrier, generally coinciding with the maximum attractive force of the pit, that needs to be overcome for cluster-merging to take place. This barrier is located at a distance of the order of the cluster radius, in agreement with experimental observations. The $P_{a}$ required for the cluster to escape the trapping force of the pit is consistent both with the measured ones and with the effective pressures that we extracted from the bubble dynamics in our previous work ${ }^{14}$. As the distance between the pits increases, the $P_{a}$ for merging also increases, up to a certain limiting distance, where the cluster cannot escape from the pits. For practical purposes, this could be regarded as an optimal distance between the pits for efficient sonochemical reactors design, where the number of the pits (i.e. of the bubbles) should be the maximum possible per unit area, but still avoiding the merging as it lowers the radical production $\underline{\underline{14}}$ and enhances the erosion of the reactor walls $\underline{\underline{16}}$.

We showed that the key to the transition to merging clusters relies on the influence of the nonlinear bubble oscillations and not on the increasing number of bubbles at increasing powers, as the $P_{a}$ for merging decreases when $N$ increases, but only until a certain 
threshold, after which it basically becomes constant. However, an indirect influence of the number of bubbles can be present, because the $P_{a}$ for transition decreases with the size of the cluster. As strongly driven bubbles tend to form stable pairs rather than to $\operatorname{coalesce}^{28}$, we can expect that increasing the number of generated bubbles will also increase the clusters radii. This dependence of the cluster dimension on the number of bubbles, as well as inertia and drag experienced by translating bubbles, should be addressed in future works, for a more complete understanding of the phenomenon.

We also showed that the bubble size has an influence on the driving pressure required for merging, which presents a minimum for clusters with bubbles of $10 \mu \mathrm{m}$. We can therefore expect that the merging and the consequent generated flow are first initiated by the bubbles of this size, with some shift towards the smaller bubbles once that also inertia and drag come into play.

Finally, we examined the sonochemical production and we found that neglecting the Bjerknes forces will lead to a huge overestimate of the number of radicals produced. This happens because the interaction with the neighbors dampens the oscillations of the bubble, reducing the temperature at collapse and also the resonance frequency. Since these interactions exhibit an inverse proportionality with the distance between the bubbles, smaller size of the clusters and shorter distances between the pits as well as higher number of bubbles strongly decrease the radical production, even before the merging takes place. This could be the key to explain the experimental observation that increasing the power after a certain threshold does not improve the sonochemical production. For practical purposes, the efficiency of a sonochemical reactor could benefit from a medium power operating condition instead of high power and a distance between the pits substantially larger, in order to prevent cluster merging, which reduces the chemical yield. 


\section{Acknowledgments}

We acknowledge innumerous discussions with Andrea Prosperetti over the years, from whom we learnt tremendously. We moreover acknowledge Technology Foundation STW and the Nederlandse Organisatie voor Wetenschappelijk Onderzoek (NWO) for financial support. 


\section{References}

1 K. S. Suslick, "Sonochemistry", Science 247, 1439-1445 (1990).

2 K. S. Suslick, S. J. Doktycz, and E. B. Flint, "On the origin of sonoluminescence and sonochemistry", Ultrasonics 28, 280-290 (1990).

${ }^{3}$ K. S. Suslick and G. J. Price, "Applications of ultrasound to materials chemistry", Ann. Rev. Mat. Sci. 29, 295-326 (1999).

4 L. A. Crum, T. J. Mason, J. L. Reisse, and K. S. Suslick, eds., See the articles in Sonochemistry and Sonoluminescence, 1-407 (Kluwer Academic Publishers, Dordrecht) (1999).

5 T. J. Mason and J. P. Lorimer, Applied sonochemistry, the uses of power ultrasound in chemistry and processing, 1-293 (Wiley-VCH, Weinheim) (2002).

${ }^{6}$ K. S. Suslick and D. J. Flannigan, "Inside a collapsing bubble: Sonoluminescence and the conditions during cavitation", Ann. Rev. Phys. Chem. 59, 659-683 (2008).

7 H. Cheung, A. Bhatnagar, and G. Jansen, "Sonochemical destruction of chlorinated hydrocarbons in diluted aqueous solutions", Environ. Sci. Technol. 25, 1510-1512 (1991).

8 A. Kotronarou, G. Mills, and M. R. Hoffmann, "Ultrasonic irradiation of paranitrophenol in aqueous-solutions", J. Phys. Chem. 95, 3630-3638 (1991).

9 A. Kotronarou, G. Mills, and M. R. Hoffmann, “Decomposition of parathion in aqueous solution by ultrasonic irradiation", Environ. Sci. Technol. 26, 1460-1462 (1992).

10 P. R. Gogate, S. Mujumdar, and A. B. Pandit, "Sonochemical reactors for waste water treatment comparison using formic acid degradation as a model reaction", Adv. Environ. Res. 7, 283-299 (2003).

11 V. G. Yechmenev, E. J. Blanchard, and A. H. Lambert, "Study of the influence of ultrasound on enzymatic treatment of cotton fabric", Text. Color. Chem. Amer. Dyestuff Rep. 1, 47-51 (1999).

12 I. Z. Shirgaonkar, R. R. Lothe, and A. B. Pandit, "Comments on the mechanism of microbial cell disruption in high pressure and high speed devices", Biotechnol. Prog. 14, 657-660 (1998). 
13 D. F. Rivas, A. Prosperetti, A. G. Zijlstra, D. Lohse, and H. J. G. E. Gardeniers, “Efficient sonochemistry through microbubbles generated with micromachined surfaces", Angew. Chem. Int. Ed. 49, 9699-9701 (2010).

14 D. F. Rivas, L. Stricker, A. Zijlstra, H. Gardeniers, D. Lohse, and A. Prosperetti, “Ultrasound artificially nucleated bubbles and their sonochemical radical production", Ultrason. Sonochem. 20, 510-524 (2013).

15 N. Bremond, M. Arora, C. D. Ohl, and D. Lohse, "Controlled multibubble surface cavitation", Phys. Rev. Lett. 96, 224501 (2006).

16 D. F. Rivas, B. Verhaagen, J. R. T. Seddon, A. G. Zijlstra, L.-M. Jiang, L. W. M. van der Sluis, M. Versluis, D. Lohse, and H. J. G. E. Gardeniers, “Localized removal of layers of metal, polymer, or biomaterial by ultrasound cavitation bubbles", Biomicrofluidics 6, 034114 (2012).

17 V. Bjerknes, Fields of forces, 30-35 (Columbia University Press, New York) (1906).

18 T. G. Leighton, The acoustic bubble, 356-366 (Academic Press, London) (1994).

19 F. G. Blake, “Onset of cavitation in liquids", Ph.D. thesis, Harvard University (1949).

${ }^{20}$ L. A. Crum, "Bjerknes forces on bubbles in a stationary sound field", J. Acoust. Soc. Am. 57, 1363-1370 (1975).

${ }^{21}$ H. N. Og̃uz and A. Prosperetti, "A generalization of the impulse and virial theorems with an application to bubble oscillations", J. Fluid Mech. 218, 143-162 (1990).

${ }^{22}$ N. A. Pelekasis and J. A. Tsamopoulos, "Bjerknes forces between two bubbles. Part 1. Response to a step change in pressure", J. Fluid Mech. 254, 467-499 (1993).

${ }^{23}$ N. A. Pelekasis and J. A. Tsamopoulos, "Bjerknes forces between two bubbles. Part 2. Response to an oscillatory pressure field", J. Fluid Mech. 254, 501-527 (1993).

24 A. A. Doinikov, "Bjerknes forces between two bubbles in a viscous fluid", J. Acoust. Soc. Am. 106, 3305-3312 (1999).

25 A. A. Doinikov, "Mathematical model for collective bubble dynamics in strong ultrasound fields", J. Acoust. Soc. Am. 116, 821-827 (2004).

${ }^{26}$ K. Yasui, Y. Iida, T. Tuziuti, T. Kozuka, and A. Towata, "Strongly interacting bubbles 
under an ultrasonic horn", Phys. Rev. E 77, 016609-1 (2008).

27 A. Prosperetti, "Bubble phenomena in sound fields: part two", Ultrasonics 22, 115-124 (1984).

28 R. Mettin, I. Akhatov, U. Parlitz, C. D. Ohl, and W. Lauterborn, “Bjerknes forces between small cavitation bubbles in a strong acoustic field", Phys. Rev. E 56, 2924-2931 (1997).

29 A. A. Doinikov, "Viscous effects on the interaction force between two small gas bubbles in a weak acoustic field", J. Acoust. Soc. Am. 111, 1602-1609 (2002).

30 A. A. Doinikov, "Translational motion of two interacting bubbles in a strong acoustic field", Phys. Rev. E 64, 026301 (2001).

31 A. Harkin, T. J. Kaper, and A. Nadim, “Coupled pulsation and translation of two gas bubbles in a liquid", J. Fluid Mech. 445, 377-411 (2001).

32 N. A. Pelekasis, A. Gaki, A. Doinikov, and J. A. Tsamopoulos, "Secondary Bjerknes forces between two bubbles and the phenomenon of acoustic streamers", J. Fluid Mech. 500, 313-347 (2004).

33 K. Yoshida, T. Fujikawa, and Y. Watanabe, “Experimental investigation on reversal of secondary Bjerknes force between two bubbles in ultrasonic standing wave", J. Acoust. Soc. Am. 130 135-144, 727-734 (2011).

34 R. Toegel, S. Hilgenfeldt, and D. Lohse, "The effect of surfactants on single bubble sonoluminescence", Phys. Rev. Lett. 84, 2509-2512 (2000).

35 R. Toegel, S. Hilgenfeldt, and D. Lohse, "Suppressing dissociation in sonoluminescing bubbles: The effect of excluded volume", Phys. Rev. Lett. 88, 034301 (2002).

36 R. Toegel and D. Lohse, "Phase diagrams for sonoluminescing bubbles: A comparison between experiment and theory", J. Chem. Phys. 118, 1863 (2003).

37 L. Stricker, A. Prosperetti, and D. Lohse, "Validation of an approximate model for the thermal behavior in acoustically driven bubbles", J. Acoust. Soc. Am. 130, 3243-3251 (2011).

38 Z. Zeravcic, D. Lohse, and W. van Saarloos, "Collective oscillations in bubble clouds", 
J. Fluid Mech. 680, 114-149 (2011).

39 L. Stricker, "Acoustic cavitation and sonochemistry", Ph.D. thesis, chap. 7, University of Twente (2012).

40 A. Prosperetti, "Bubble phenomena in sound fields: part one", Ultrasonics 22, 69-77 (1984).

${ }^{41}$ H. Gelderblom, A. G. Zijlstra, L. van Wijngaarden, and A. Prosperetti, “Oscillations of a gas pocket on a liquid-covered solid surface", Phys. Fluids 24, 122101 (2012).

42 A. Zijlstra, "Acoustic surface cavitation", Ph.D. thesis, p. 77, University of Twente (2011).

${ }^{43}$ M. S. Plesset, "Comment on 'Sonoluminescence from water containing dissolved gases' (J. Acoust. Soc. Am. 60, 100-103 (1976))", J. Acoust. Soc. Am. 62, 470 (1977).

44 M. S. Plesset and A. Prosperetti, "Bubble dynamics and cavitation", Annu. Rev. Fluid Mech. 9, 145-185 (1977). 


\section{List of Figures}

FIG. 1 Example of the three different behaviors of the clusters observed in experiments, at increasing applied power: clusters close to the pits from which their bubbles originated (behavior 1, top), clusters pointing toward the center (behavior 2, middle), and clusters migrating toward the center (behavior 3, bottom). The left column was recorded at normal speed and represents therefore a time average, while the right column shows single snapshots taken with $7 \mathrm{~ns}$ exposure time. The experimental conditions are the same described in Ref. $\underline{14} . \ldots$. . . . . . . . . . . . . . . . . . .

FIG. 2 Sketch of the secondary Bjerknes forces acting upon the bubble and introduction of our employed notation. $F_{c}$ is the force from cluster $2, F_{p 1,2}$ are forces from the pits and $F_{m 1,2}$ are the forces from the mirror clusters. . . .

FIG. 3 Forces acting upon a bubble of cluster 1 with an initial displacement $\delta=$ $R_{0}$ respect to its unperturbed condition. Parameters: $R_{c}=100 \mu \mathrm{m}, N=$ $100, d=1000 \mu \mathrm{m}, R_{0}=10 \mu \mathrm{m}, f=200 \mathrm{kHz}$. The cluster-cluster forces are always attractive, while the pit-cluster forces become repulsive at high driving, thus favoring the inception of the motion. . . . . . . . . .

FIG. 4 Average forces $\left\langle F_{x}^{*}\right\rangle$ (solid) and $\left\langle F_{p 1, x}\right\rangle$ (dash-dot) acting upon a bubble of cluster 1, over one acoustic cycle, as function of $\delta$ for $R_{0}=10 \mu \mathrm{m}, N$ $=100, R_{c}=100 \mu \mathrm{m}, d=1000 \mu \mathrm{m}, f=200 \mathrm{kHz}$. In lit-gray, low pressure case: $\left\langle F_{x}^{*}\right\rangle$ is too weak to pass the barrier constituted by the restoring force $\left\langle F_{p 1, x}\right\rangle$; the cluster remains attached to its pit, with a small displacement given by the first intersection of the two curves. In black, high pressure case: $\left\langle F_{x}^{*}\right\rangle>\left\langle F_{p 1, x}\right\rangle$, and the two clusters merge. In mid-gray, critical case, defining the transition between both behaviors. $\bar{\delta}$ represents the maximum displacement of the cluster before the transition. . . . . . . . . 
FIG. 5 Calculated driving pressure for transition from behavior 1 to 2, i.e. the inception of the motion (dash) and from behavior 2 to 3, i.e. the merging of the clusters (solid), as function of the distance between the pits for a cluster with $R_{0}=10 \mu \mathrm{m}, N=100, R_{c}=100 \mu \mathrm{m}$, driven at $f=200 \mathrm{kHz}$. When the pits are too far apart $(d \gtrsim 1350 \mu \mathrm{m})$, no merging is possible. . .

FIG. 6 Electric potential applied to the piezo when the clusters start to merge, at increasing power (black), and when they detach going back to their own pits, at decreasing power (gray), as function of the distance between the pits, in the experiment described in Ref. $\underline{\underline{14}}$. Increasing voltage corresponds to increasing $P_{a}$. For $d>1500 \mu \mathrm{m}$ no merging was observed, no matter $P_{a}$.

FIG. 7 Driving pressure (solid) and maximum displacement of the cluster (dashdot) at transition from behavior 2 to behavior 3, as function of the number of bubbles. Parameters: $R_{0}=10 \mu \mathrm{m}, R_{c}=100 \mu \mathrm{m}, d=1000 \mu \mathrm{m}, f$ $=200 \mathrm{kHz}$. For $N>50$ they become both almost invariant to a further increase of $N \ldots \ldots \ldots \ldots \ldots \ldots \ldots \ldots \ldots \ldots \ldots \ldots$

FIG. 8 Driving pressure (solid) and maximum displacement of the cluster (dashdot) at transition from behavior 2 to behavior 3, as function of the radius of the cluster. Parameters: $R_{0}=10 \mu \mathrm{m}, N=100, d=1000 \mu \mathrm{m}, f=200 \mathrm{kHz}$. For bigger clusters the $P_{a}$ required for merging is lower, as well as the maximum displacement of the cluster. . . . . . . . . . . . . . .

FIG. 9 Driving pressure (solid) and maximum displacement of the cluster (dashdot) at transition from behavior 2 to behavior 3 , as function of the radius of the bubbles. Parameters: $R_{c}=100 \mu \mathrm{m}, N=100, d=1000 \mu \mathrm{m}, f=200 \mathrm{kHz}$. The $P_{a}$ required for merging presents a minimum with bubbles of $10 \mu \mathrm{m}$, as well as the maximum displacement, which is however almost invariant

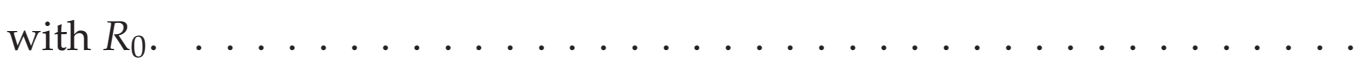


FIG. 10 Radius vs time (top) and temperature vs time (bottom) curves, for a bubble belonging to cluster 1 . The driving amplitude corresponds to the calculated one for the transition between behavior 2 and behavior 3, both including (solid) and disregarding (dash-dot) the Bjerknes forces for $P_{a}=$ $270 \mathrm{kPa}$. The dashed lines correspond to an isolated bubble, driven at $P_{a}$ $=160 \mathrm{kPa}$, that equals the "effective pressure" deduced from the bubble dynamics disregarding Bjerknes forces, just before transition in Ref. $\stackrel{\underline{14}}{\text {. Pa- }}$ rameters: $R_{0}=3 \mu \mathrm{m}, R_{c}=100 \mu \mathrm{m}, N=100, f=200 \mathrm{kHz}, d=1000 \mu \mathrm{m}, a=$

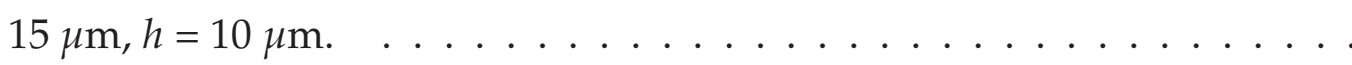

FIG. 11 Number of $\mathrm{OH}$ radicals produced as function of time, for a bubble belonging to cluster 1 driven at $P_{a}=270 \mathrm{kPa}$, with (solid) and without (dash-dot) Bjerknes forces, and at $P_{a}=160 \mathrm{kPa}$ without Bjerknes forces (dash). Driving conditions and dimensions as in Fig. $10, \ldots \ldots \ldots \ldots$

FIG. 12 Maximum number of $\mathrm{OH}$ radicals in one acoustic cycle for a bubble of cluster 1 in the unperturbed position $(\delta=0 \mu m)$ as function of the distance between the pits, for different cluster sizes (as indicated in the legend). Parameters: $N=100, R_{0}=3 \mu \mathrm{m}, f=200 \mathrm{kHz}, P_{a}=170 \mathrm{kPa} . \ldots \ldots$

FIG. 13 Maximum number of $\mathrm{OH}$ radicals in one acoustic cycle for a bubble of cluster 1 in the unperturbed position $(\delta=0 \mu \mathrm{m})$ as function of the number of bubbles. Parameters: $R_{c}=100 \mu \mathrm{m}, d=1000 \mu \mathrm{m}, R_{0}=3 \mu \mathrm{m}, f=200 \mathrm{kHz}$,

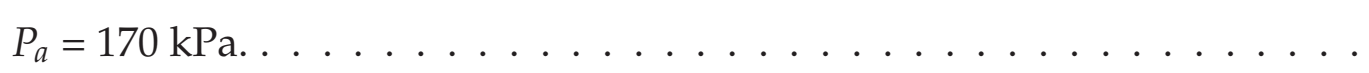




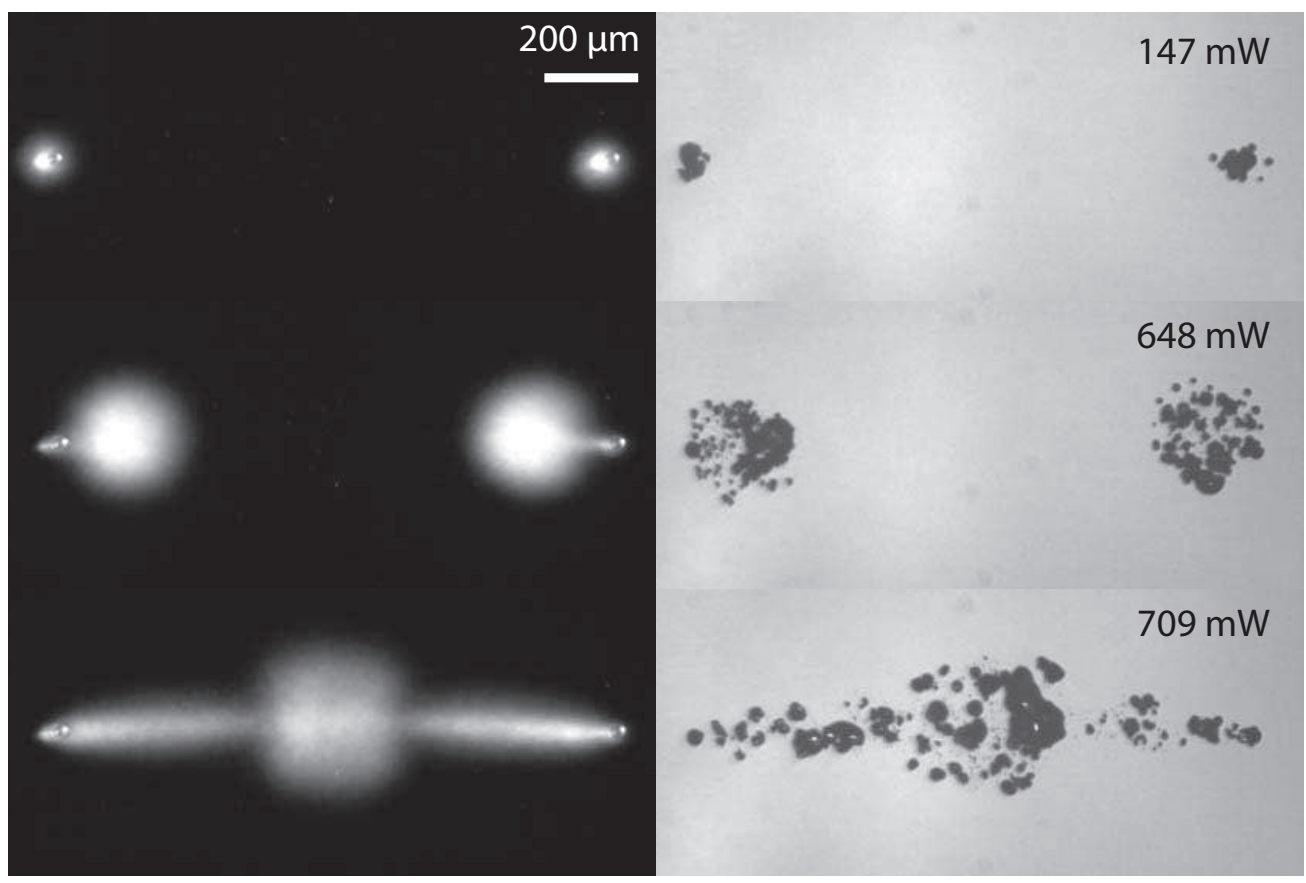

FIG. 1.

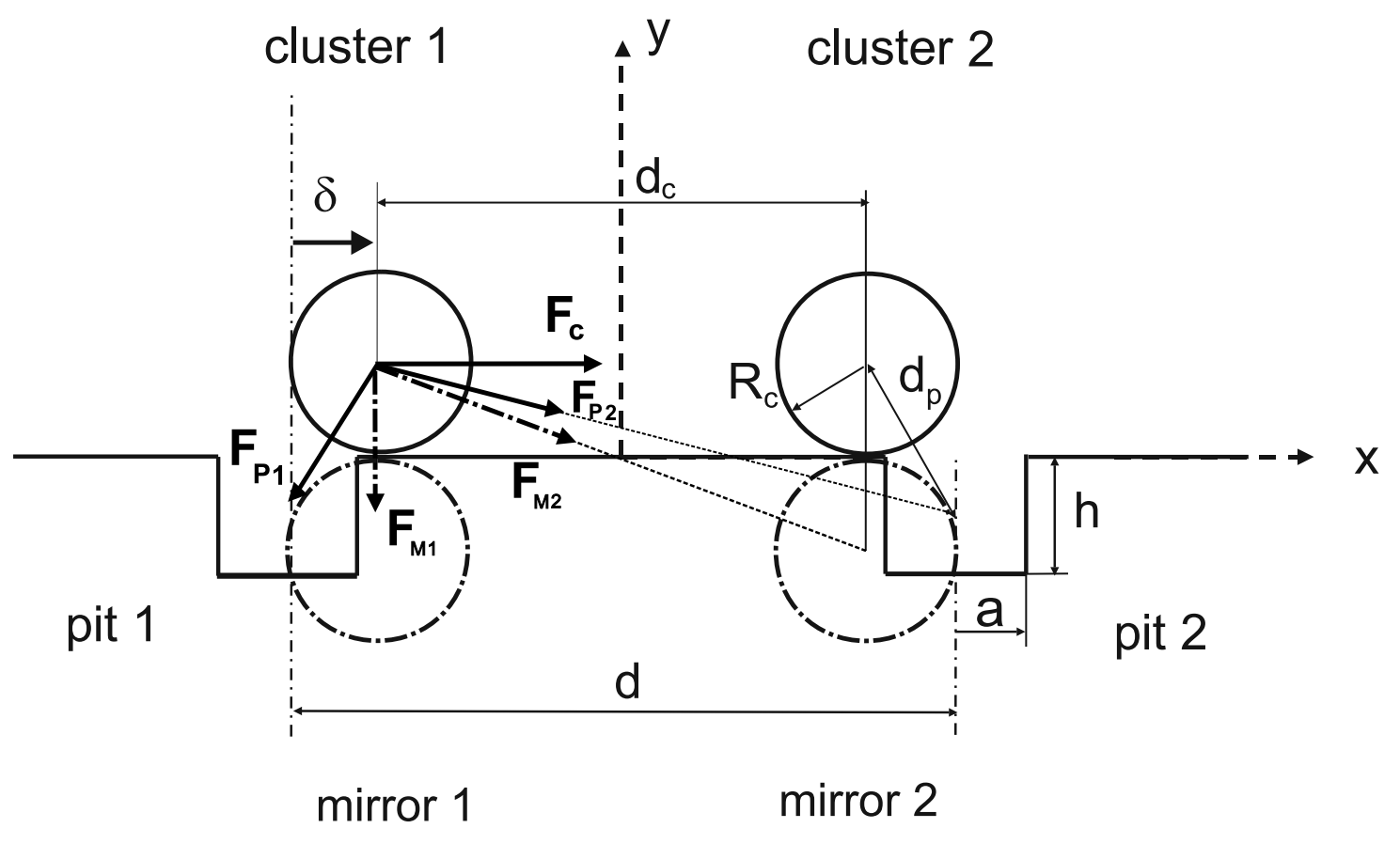

FIG. 2. 


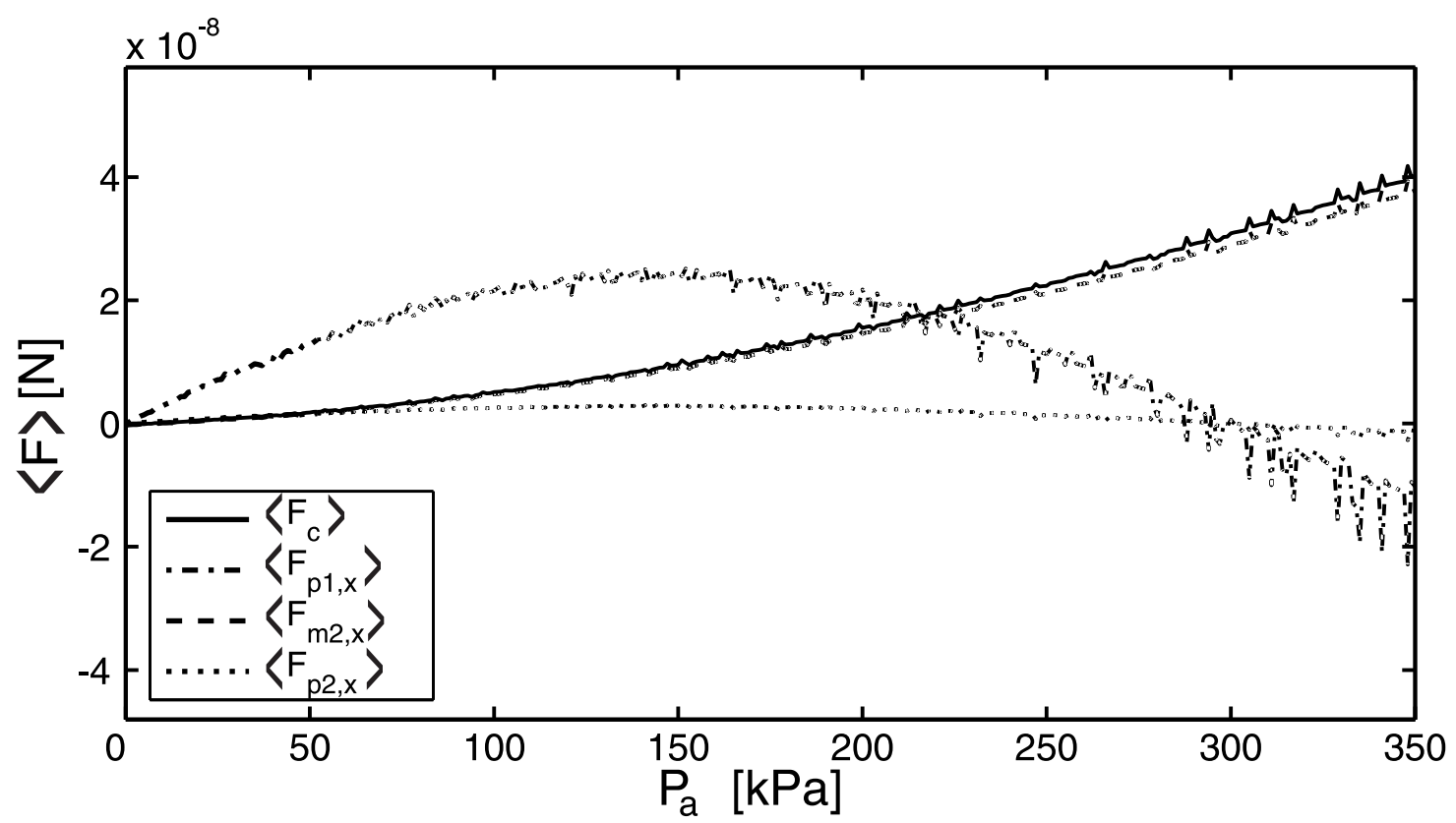

FIG. 3.

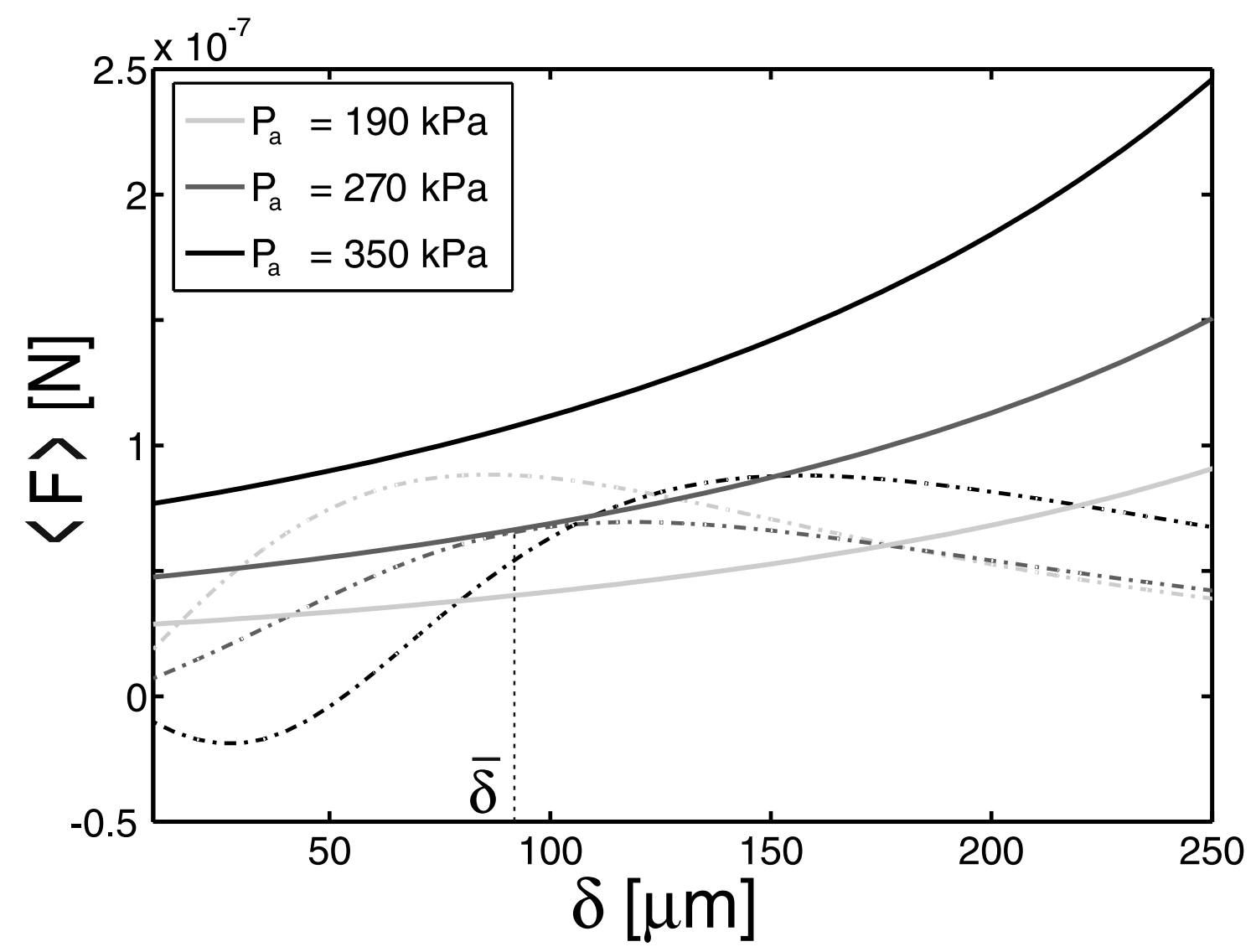

FIG. 4. 


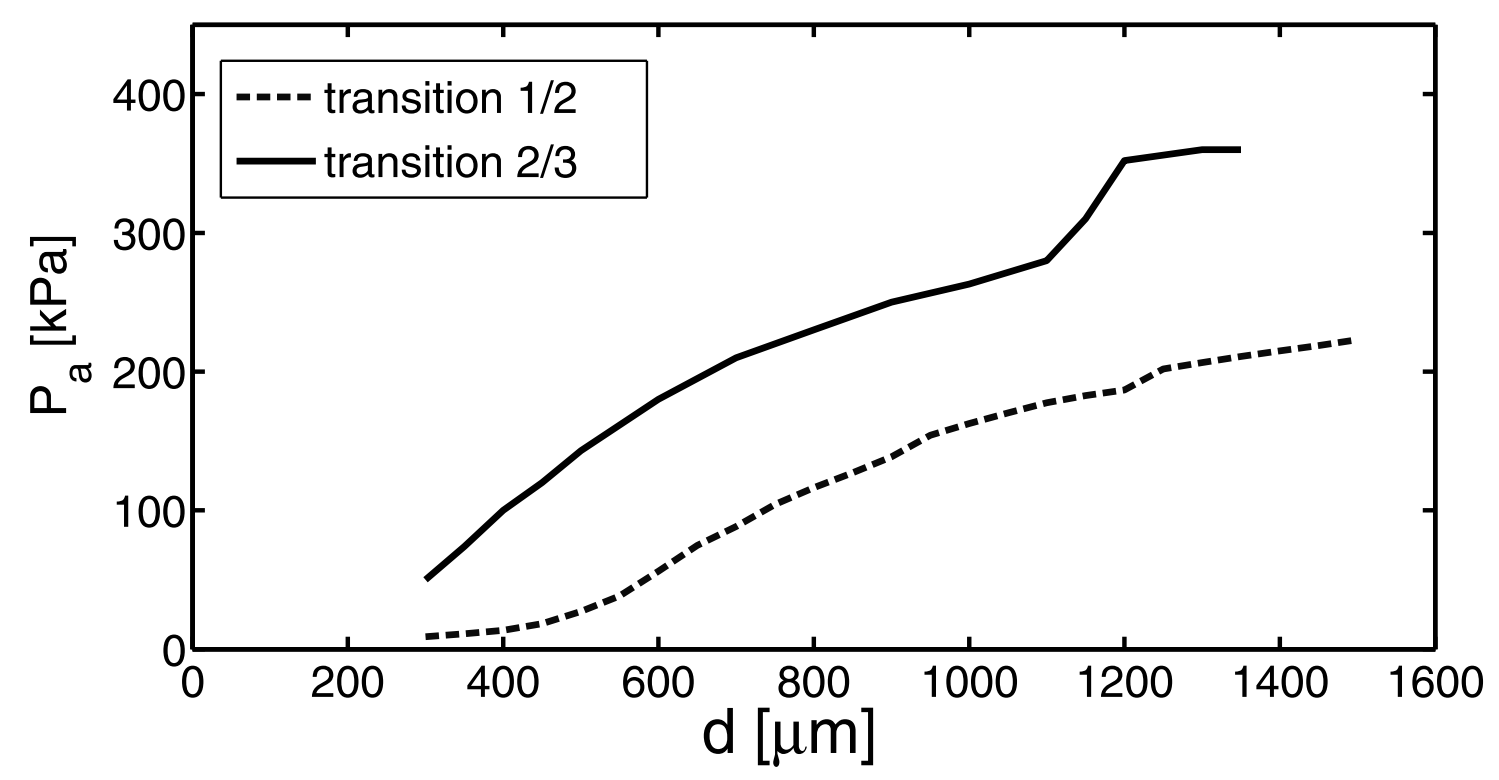

FIG. 5.

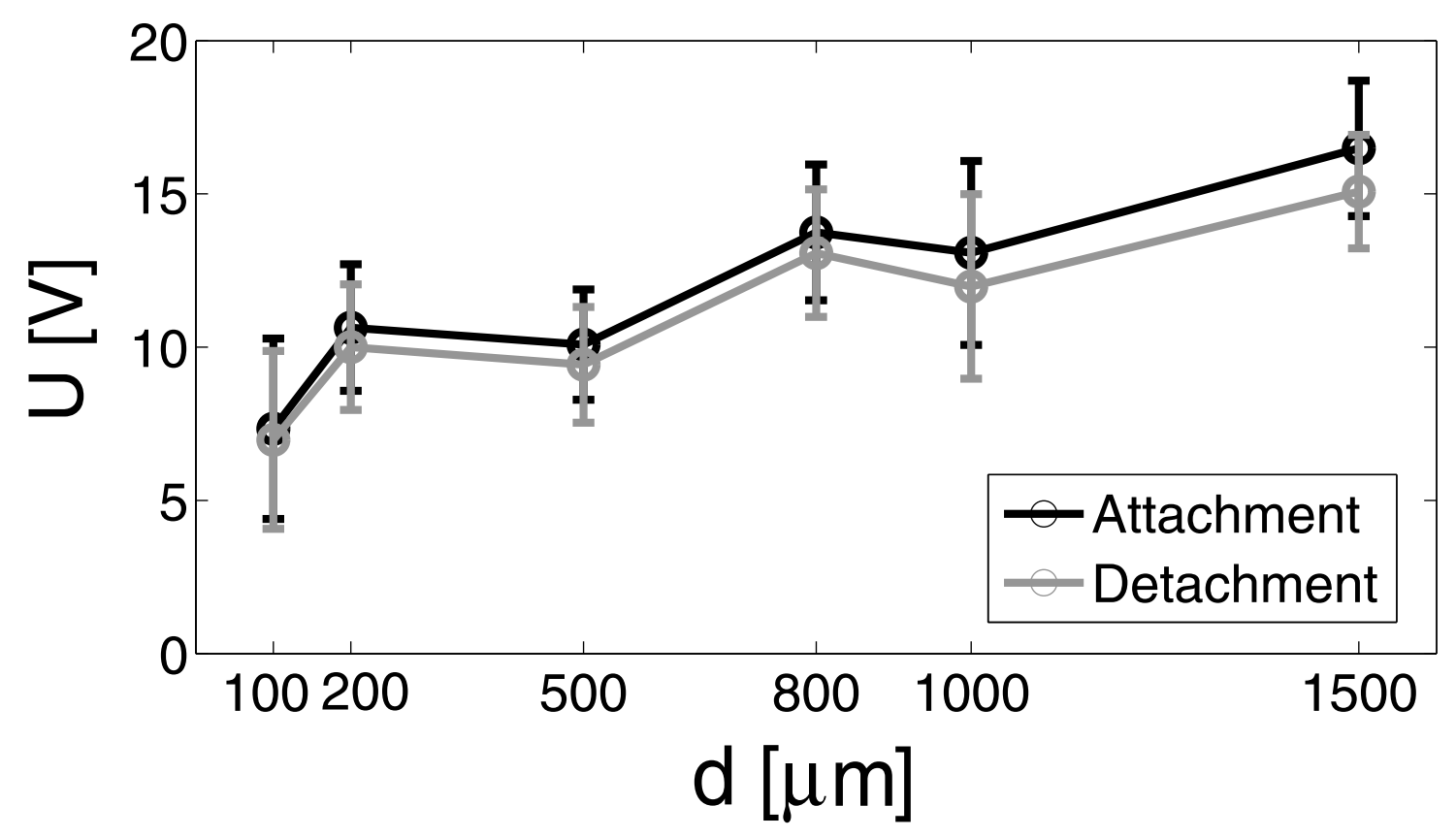

FIG. 6. 


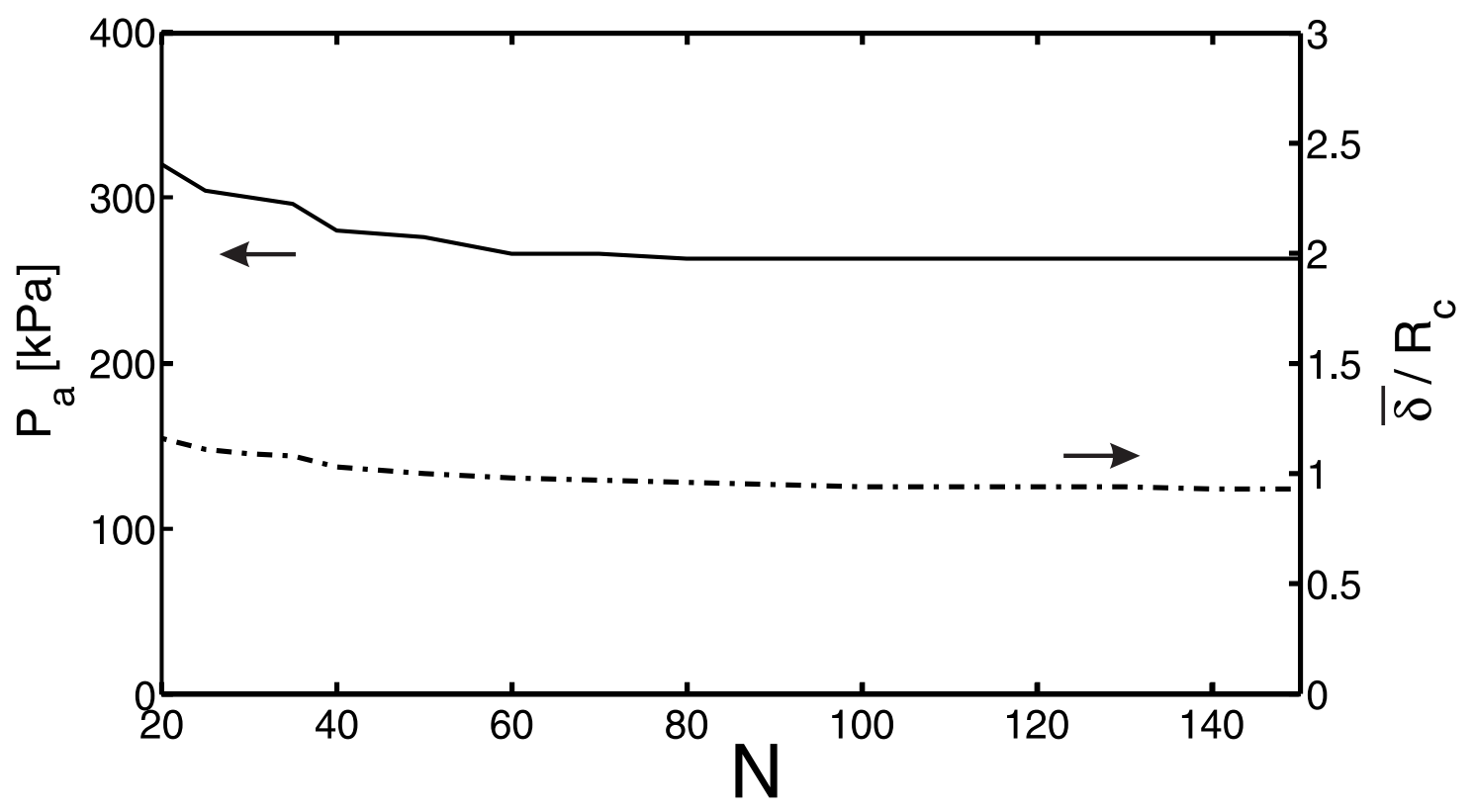

FIG. 7.

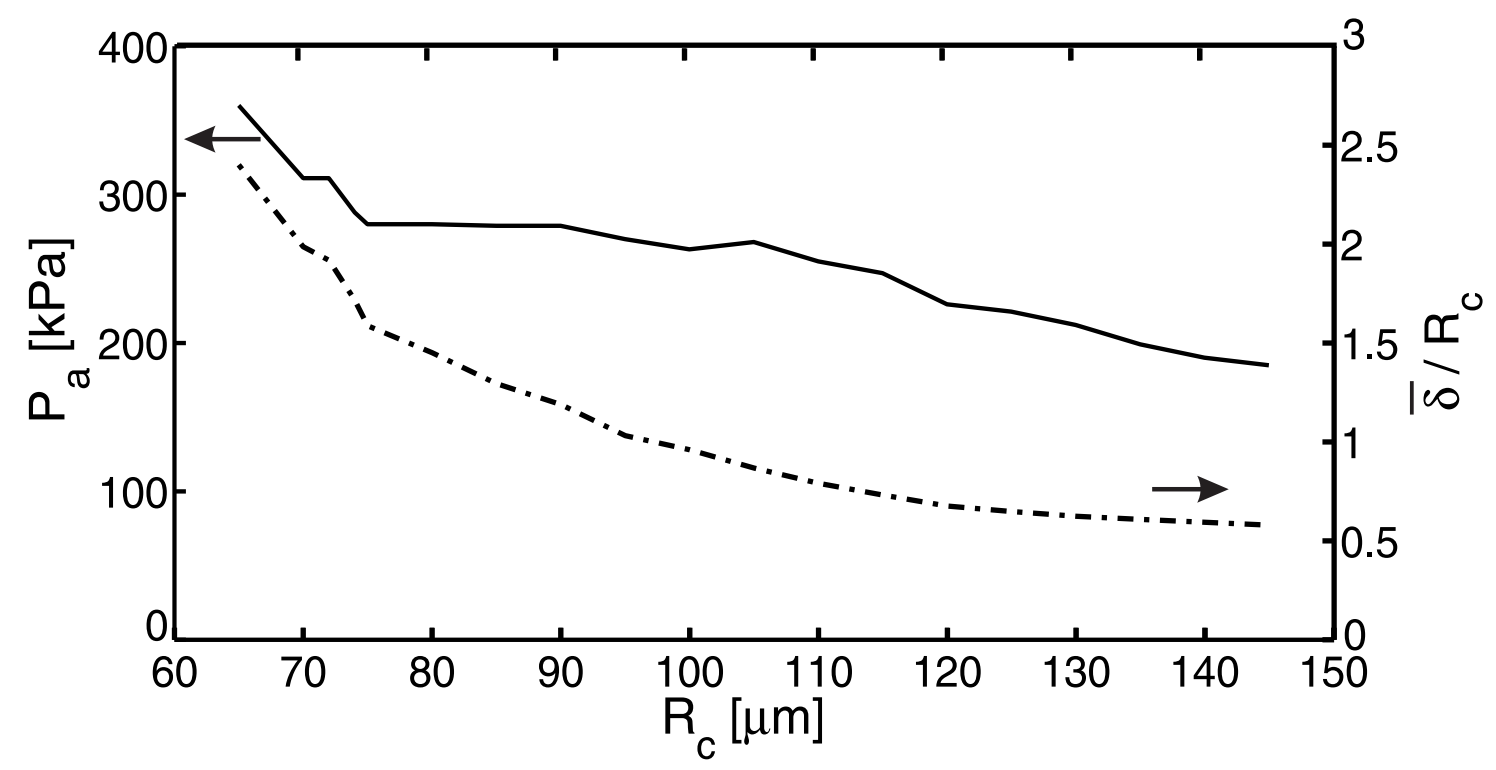

FIG. 8. 


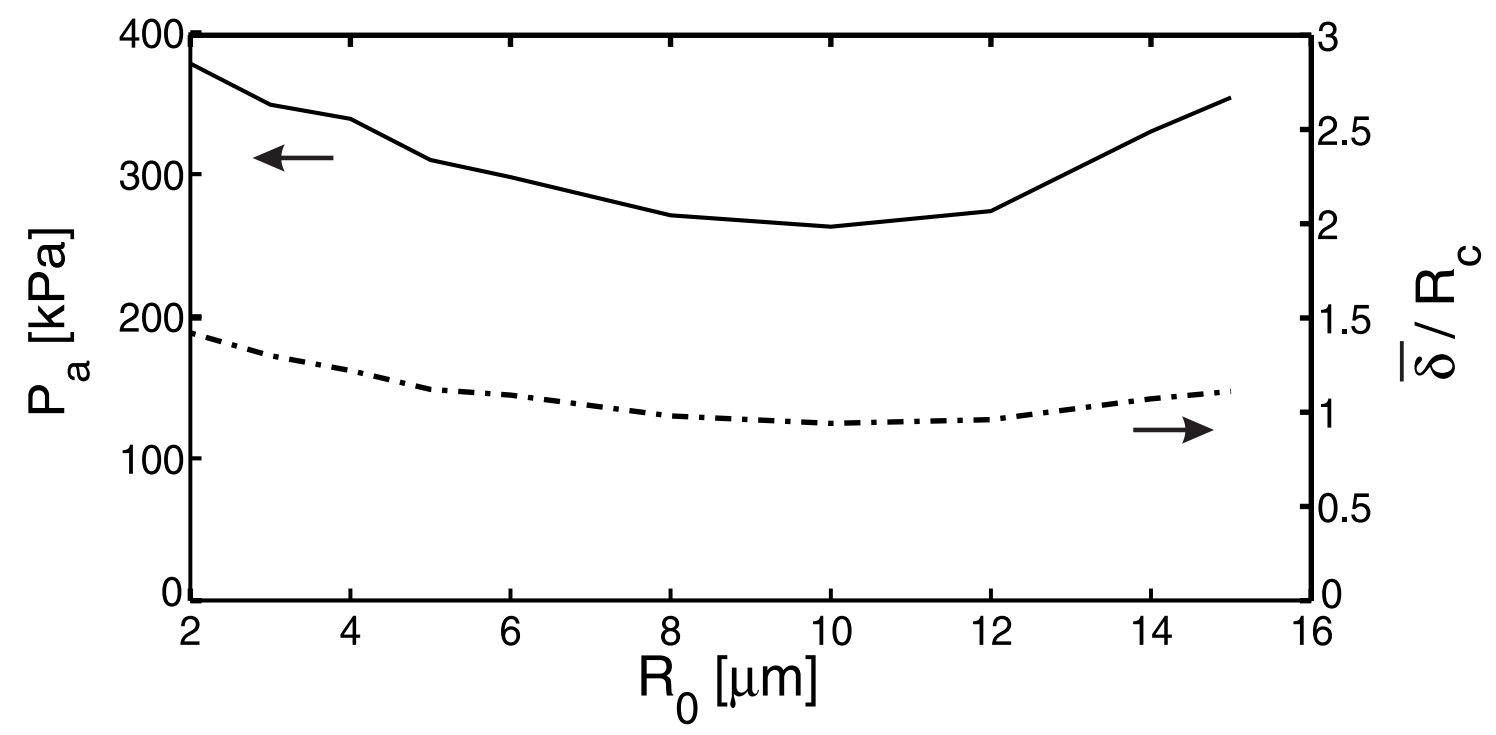

FIG. 9. 


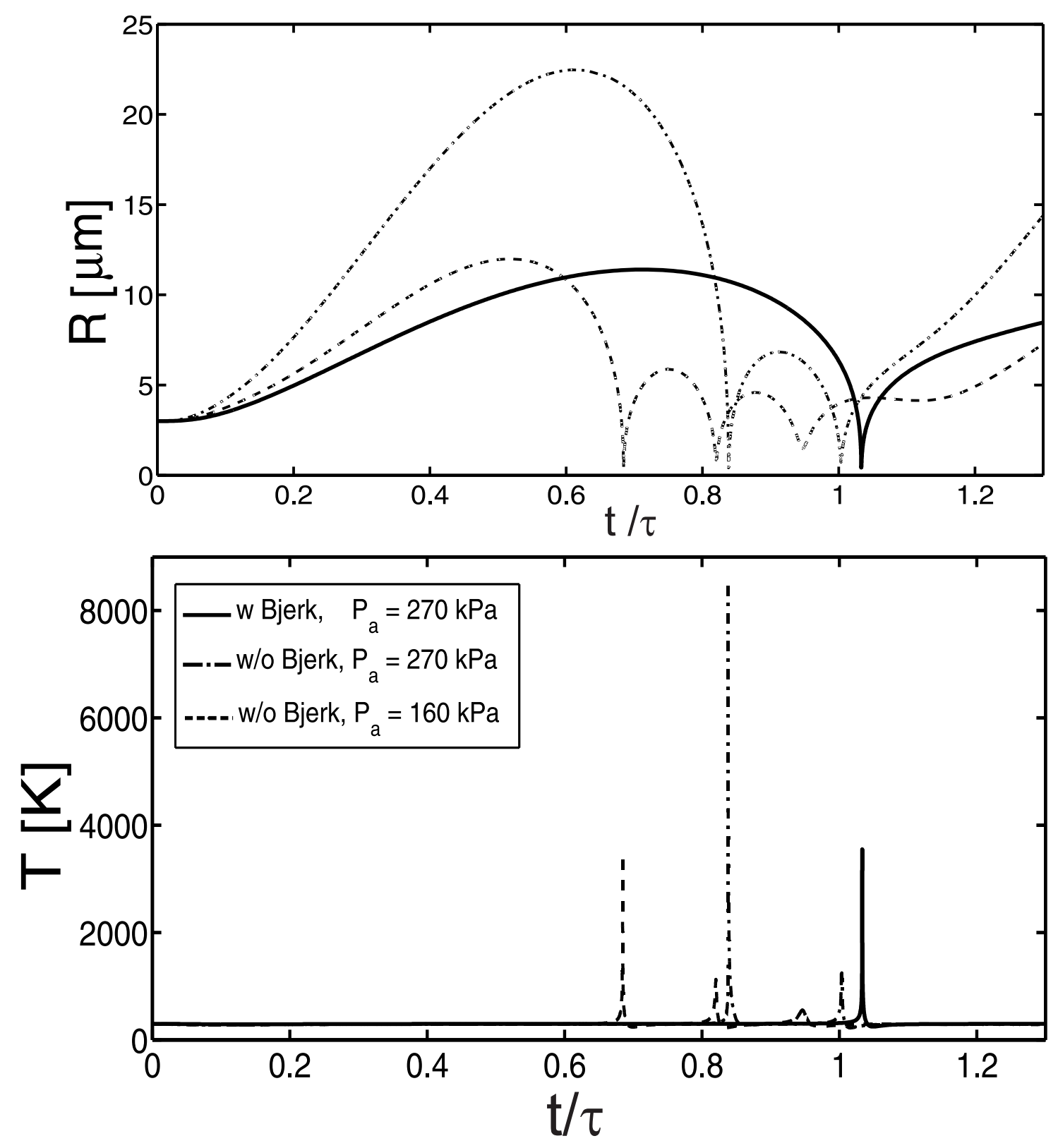

FIG. 10. 


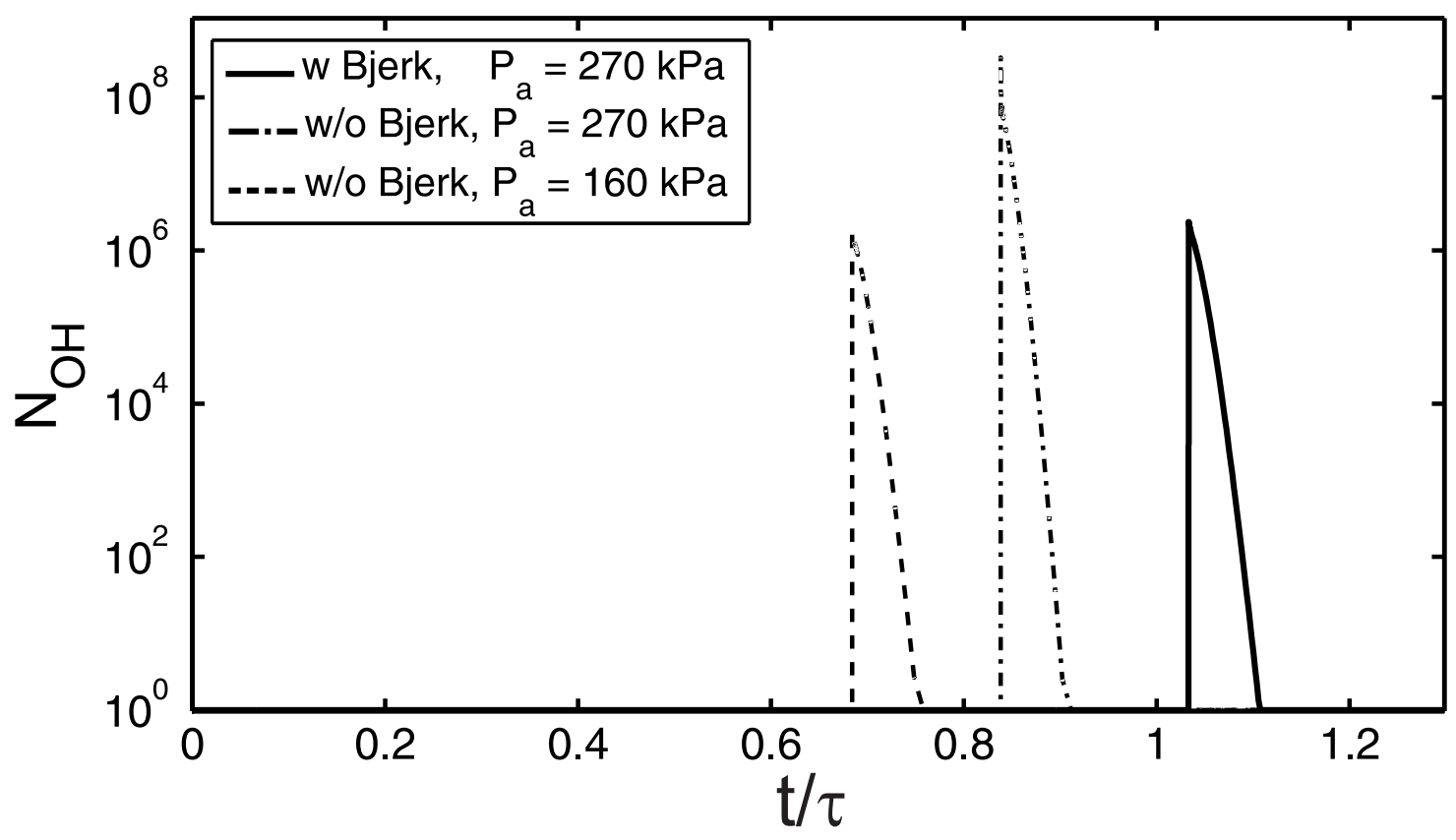

FIG. 11.

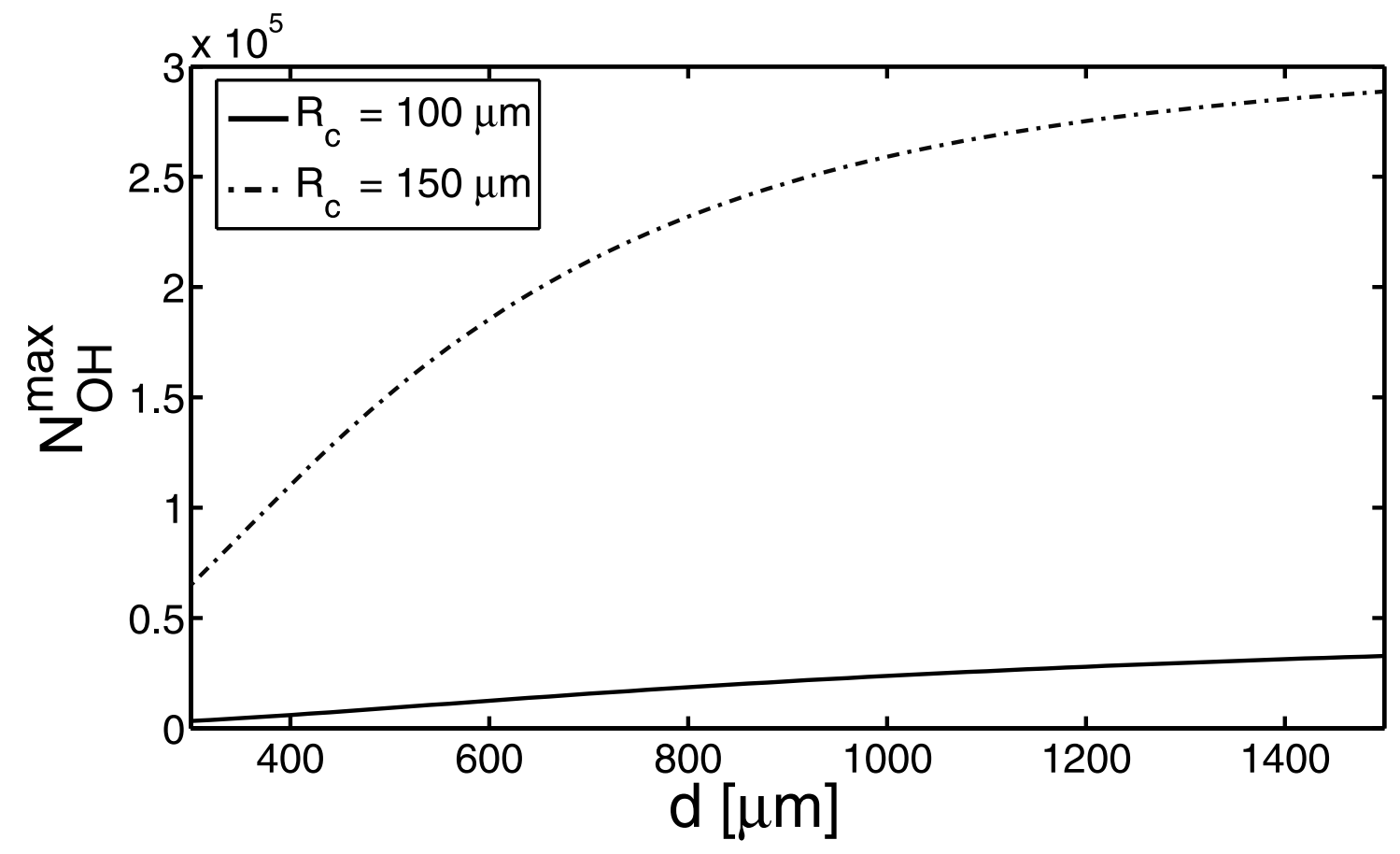

FIG. 12. 


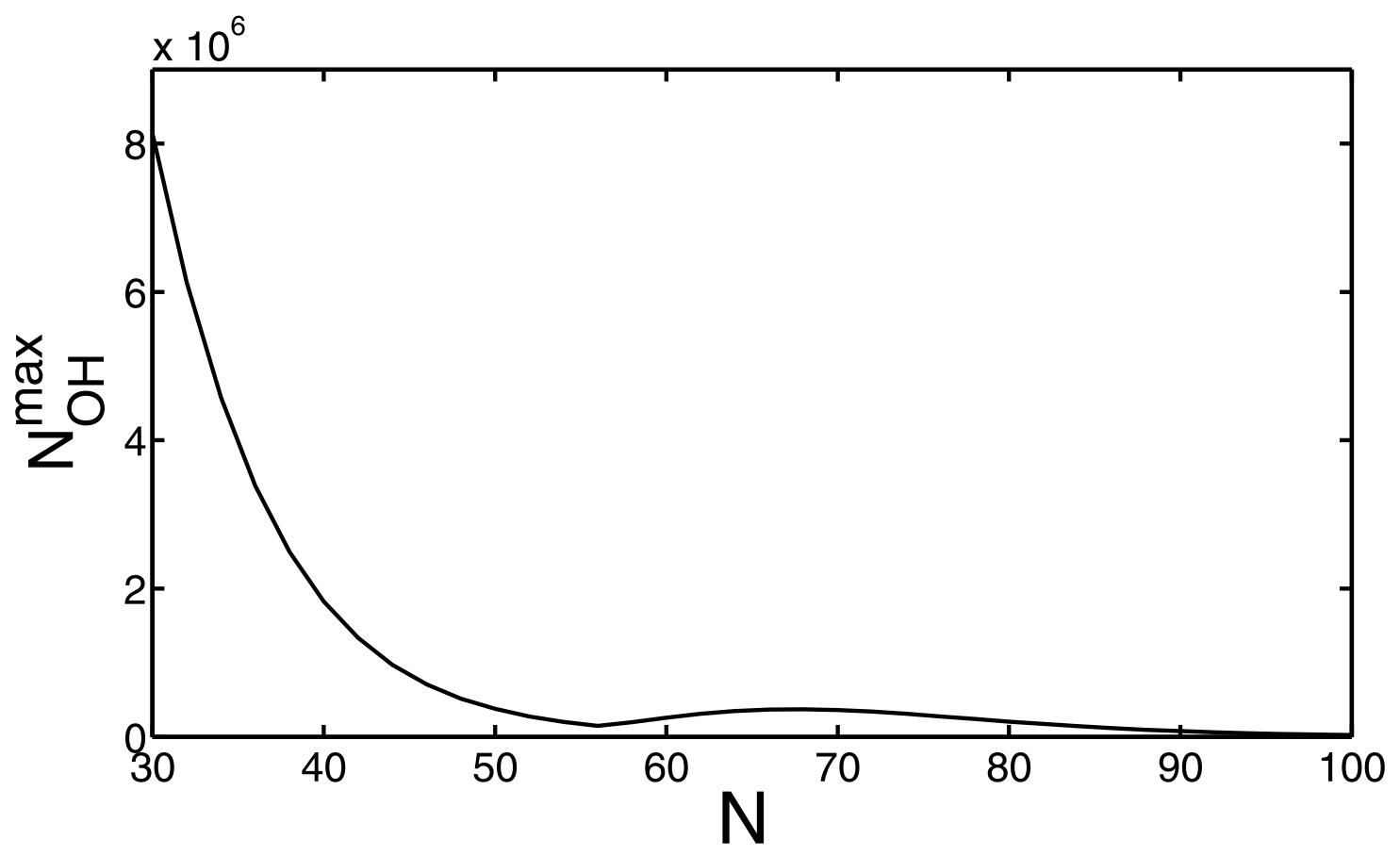

FIG. 13. 